\title{
Rehabilitation robotics research at the National Institute on Disability and Rehabilitation Research
}

\section{INTRODUCTION}

The application of robotics to disability and rehabilitation research is fairly recent. This field of research appears to be growing. The National Library of Medicine's MEDLINE is the largest freely accessible public database of medical literature, with more than 11 million citations from 1960 to the present (http://www.ncbi.nlm.nih.gov/entrez/query.fcgi). The number of publications cited each year in MEDLINE under the medical subject heading "robotics" was minimal until about 1985; after 1985 the number of citations each year began to increase. Between 1960 and 2004, 148 citations were specific to disability and rehabilitation out of 2,957 total robotics citations in MEDLINE. The research productivity in robotics over time is illustrated in Figure 1.

Robotics research productivity specific to disability and rehabilitation (Figure 1, left $y$-axis) appears to have paralleled, at a reduced level, the research productivity in all other aspects of medical robotics (Figure 1, right $y$-axis).

The Institute of Electrical and Electronics Engineers (IEEE) International Conference on Rehabilitation Robotics meets every 2 years. Their ninth

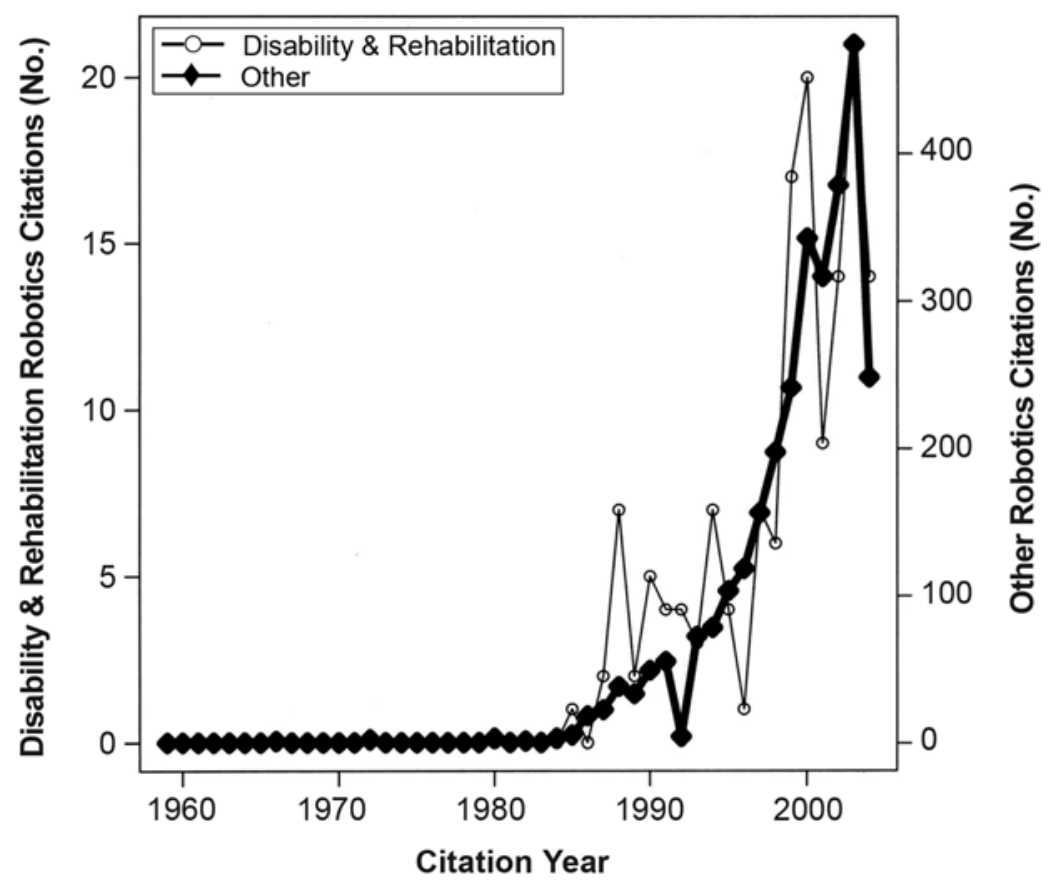

Figure 1.

MEDLINE robotics citations from 1960 to 2004. Apparent drop in 2004 publications reflects fact that MEDLINE search was performed in fall 2004. 
conference was held in Chicago, Illinois, from June 28 to July 1, 2005. Thus, the emergence of this professional meeting roughly coincides with the onset of increased publications as documented by MEDLINE. Publication of rehabilitation robotics research in the IEEE Transactions on Neural Systems and Rehabilitation Engineering has also paralleled the trend seen in MEDLINE.

\section{REVIEW OF RESEARCH FUNDED BY NATIONAL INSTITUTE ON DISABILITY AND REHABILITATION RESEARCH}

An archive of research funded by the National Institute on Disability and Rehabilitation Research (NIDRR) from 1987 forward is available in the NIDRR Program Directory (http://www.naric.com/ research/pd/). NIDRR funding of rehabilitation robotics projects was compiled from this directory and is illustrated in Figure 2.

However, determining the exact funding level for 2002 to 2004 (Figure 2) was difficult, since two of the research centers included in these data also performed research in areas other than robotics. NIDRR funding mechanisms for rehabilitation robotics include large center grants (Rehabilitation Engineering Research Centers [RERCs]), Small Business Innovation Research (SBIR) grants, and Field Initiated Project (FIP) grants. The funding data shown in Figure 2 are based on Federal fiscalyear data and do not include no-cost extensions or supplemental grant funds.

NIDRR's first rehabilitation robotics grant was awarded in 1988 to Gaulladet University (Washington, DC) for the project "Robotic finger spelling hand for communication and access to text by deafblind persons." In this project, the researchers developed and tested a prototype robotic hand. This device was never commercialized, but given the advances in robotic technology since 1988, the concept remains relevant for future research.

Since 1987, NIDRR has funded two RERCs in rehabilitation robotics. The first RERC was at the Alfred I. duPont Hospital for Children (Wilmington,

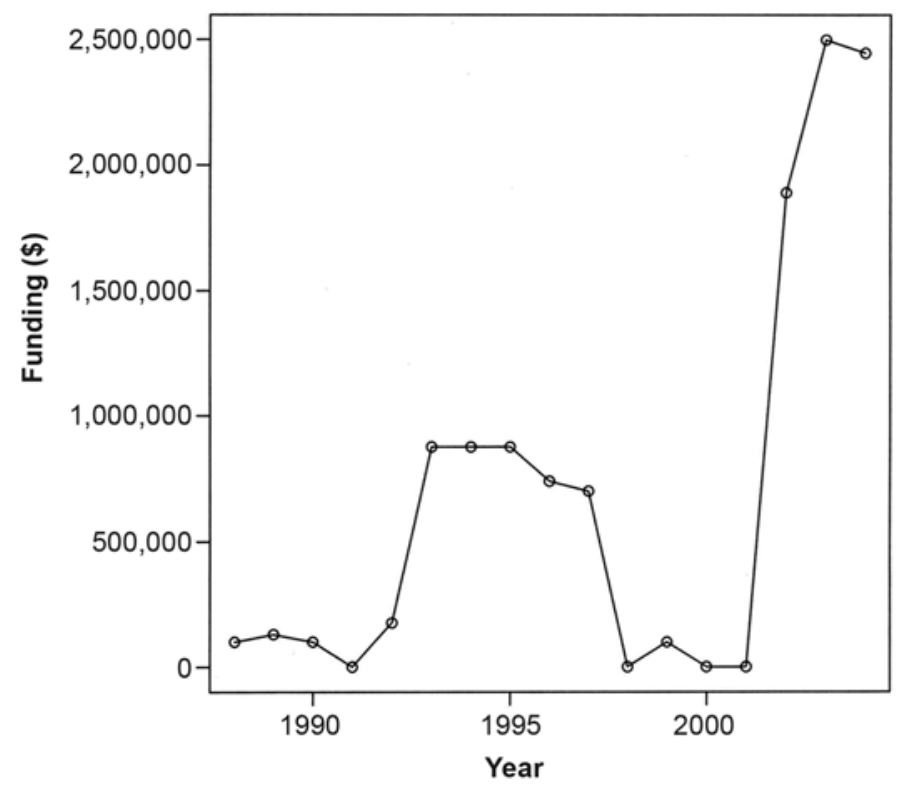

Figure 2.

National Institute on Disability and Rehabilitation Research (NIDRR) funding levels for rehabilitation robotics from 1987 to 2004. Data are based on amounts published in annual NIDRR Program Directory (http://www.naric.com/research/pd/) and do not reflect grant supplements and no-cost extensions. Data from 2002 to 2004 are estimated because some grants funded during this period had subprojects on robotics in addition to other research topics.

Delaware) and was funded from 1993 to 1997 . The second RERC at the Rehabilitation Institute of Chicago (Chicago, Illinois) received funding in 2002 for a 5-year period.

The RERC on Rehabilitation Robotics to Enhance the Functioning of Individuals with Disabilities at the Applied Science and Engineering Laboratories, Alfred I. duPont Hospital for Children, and the University of Delaware focused on interfaces, design and application, and motor control of rehabilitation robotics, as well as related information and dissemination. Within its research focus, this RERC conducted interdisciplinary research and information projects. Research and information activities were constituent-oriented and included the implementation of a consumer innovation laboratory. This laboratory involved the consumers (potential users) in the engineering design and fabrication of robotic devices for people with disabilities.

The RERC on Rehabilitation Robotics and Telemanipulation: Machines Assisting Recovery from Stroke (MARS) at the Rehabilitation Institute of 
Chicago centers its research and development on the restoration of function in hemispheric stroke survivors. Five RERC projects assess different approaches to improving upper-limb performance: (1) robot-aided therapy for force training the upper limb in people with chronic hemiparetic stroke, (2) goal-directed, robot-aided treadmill training for gait restoration in people with hemiparetic stroke, (3) development of a robotic system with an augmented reality interface for rehabilitation of people with brain injuries, (4) rehabilitation of finger extension in people with chronic hemiplegia, and (5) development of a home-based telerehabilitation system for improving functional recovery of hand and arm movement following stroke. In addition to these projects, this RERC is developing tools for training client populations, including medical students, physician residents, graduate students in engineering and neuroscience, and allied health clinicians, including physical and occupational therapists, in robotics research and development. The RERC's broad intent is to develop devices that will help the therapist provide rationally based, intensive, and long-duration treatments. The Rehabilitation Institute of Chicago collaborates with the National Rehabilitation Hospital (Washington, DC), The Catholic University of America (Washington, DC), the University of Illinois at Chicago (Chicago, Illinois), and the University of California, Irvine (Irvine, California).

Two other NIDRR-funded projects have subprojects in robotics. At the University of Pittsburgh Traumatic Brain Injury Model System (Pittsburgh, Pennsylvania), one such subproject is studying innovation in rehabilitation technology for persons with traumatic brain injury (TBI). In collaboration with The Robotics Institute at Carnegie Mellon University (Pittsburgh, Pennsylvania), these researchers are conducting a randomized trial to evaluate the efficacy of virtual reality and robotics for persons with TBI. The other NIDRR subproject, "Selective dynamic strength training to enhance upper-limb coordination following stroke," at Northwestern University (Evanston, Illinois) is investigating a novel selective dynamic strengthening protocol that incorporates a two-joint robotic device for overcoming gravity-induced abnormal movement synergies following hemiparetic stroke. The specific objectives of this project include the evaluation and demonstration of the usefulness and effectiveness of this selective dynamic robot-aided training regime in enhancing stroke survivors' quality of life. The longterm objectives of this project are the technological development and implementation of a simple, userfriendly, robot-aided therapeutic intervention that is accessible to consumers in both in- and outpatient clinics.

\section{FUTURE CONSIDERATIONS}

NIDRR's research investments in rehabilitation robotics support President George W. Bush's New Freedom Initiative (NFI), which was announced on February 1, 2001. The NFI was designed to break down the barriers to equality that continue to face Americans with disabilities and further the progress made since the passage of the Americans with Disabilities Act of 1990. One of the broad tenets of the NFI is increased access to assistive and universally designed technologies for individuals with disabilities.

Predicting future NIDRR funding levels for specific areas of research, such as rehabilitation robotics, is difficult given budget uncertainty and competing priorities. NIDRR remains committed to funding only the highest-quality peer-reviewed research. NIDRR's FIP and SBIR programs will continue to allow applications in disability and rehabilitation research, including rehabilitation robotics. Larger NIDRR centers, such as RERCs, are likely to continue along the lines of proposed NIDRR research priorities that are published periodically in the Federal Register for public comment, and followed by subsequent final notice and an invitation for investigators to submit grant applications that respond to the research priorities.

\section{CONCLUSIONS}

NIDRR-funded research appears to be moving from a paradigm of building robotic arms with which persons with disabilities can perform activities of 
daily living to a paradigm of developing robotic devices that assist with therapy in the hopes of achieving longer-term performance gains. NIDRR continues to monitor all fields of research, including rehabilitation robotics, for emerging trends and rapid advances in specific areas. NIDRR and its grantees continuously strive to produce research outcomes that will ultimately assist individuals with disabilities.

\section{ACKNOWLEDGMENT}

Dr. Jaeger was on staff at NIDRR, Office of Special Education and Rehabilitative Services, U.S.
Department of Education, Washington, DC, from 1997-2006.

\section{Robert J. Jaeger, PhD}

Program Director, Research to Aid Persons with Disabilities Program; Chemical, Bioengineering, Environmental, and Transport Systems Division; National Science Foundation, Arlington, VA

Email: rjaeger@nsf.gov

DOI: 10.1682/JRRD.2006.05.0041 